\title{
Isolation and screening of potential actinobacteria for rapid composting of rice straw
}

\begin{abstract}
Rice straw is produced as a by-product from rice cultivation, which is composed largely of lignocellulosic materials amenable to general biodegradation. Lignocellulolytic actinobacteria can be used as a potential agent for rapid composting of bulky rice straw. Twenty-five actinobacteria isolates were isolated from various in situ and in vitro rice straw compost sources. Isolates A2, A4, A7, A9 and A24 were selected through enzymatic degradation of starch, cellulose and lignin followed by the screening for their adaptability on rice straw powder amended media. The best adapted isolate (A7) was identified as Micromonospora carbonacea. It was able to degrade cellulose, hemicelluloses and carbon significantly (P Ò 0.05 ) over the control. $\mathrm{C} / \mathrm{N}$ ratio was reduced to 18.1 from an initial value of 29.3 in 6 weeks of composting thus having the potential to be used in large scale composting of rice straw.
\end{abstract}

Keyword: Actinobacteria; Biodegradation; Cellulases; Ligninolytic enzymes; Micromonospora carbonacea; Rice straw 